\title{
Schimke immunoosseous dysplasia: defining skeletal features
}

\author{
Kshamta B. Hunter • Thomas Lücke • Jürgen Spranger • Sarah F. Smithson • \\ Harika Alpay • Jean-Luc André • Yumi Asakura • Radovan Bogdanovic • \\ Dominique Bonneau • Robyn Cairns $\cdot$ Karlien Cransberg • Stefan Fründ • \\ Helen Fryssira • David Goodman • Knut Helmke • Barbara Hinkelmann • \\ Guiliana Lama • Petra Lamfers • Chantal Loirat - Silvia Majore • Christy Mayfield • \\ Bertram F. Pontz • Cristina Rusu • Jorge M. Saraiva • Beate Schmidt • \\ Lawrence Shoemaker $\cdot$ Sabine Sigaudy $\cdot$ Natasa Stajic $\cdot$ Doris Taha $•$ \\ Cornelius F. Boerkoel
}

Received: 10 September 2009 / Accepted: 17 November 2009/Published online: 15 December 2009

(C) The Author(s) 2009. This article is published with open access at Springerlink.com

\begin{abstract}
Schimke immunoosseous dysplasia (SIOD) is an autosomal recessive multisystem disorder characterized by prominent spondyloepiphyseal dysplasia, T cell deficiency, and focal segmental glomerulosclerosis. Biallelic mutations

Electronic supplementary material The online version of this article (doi:10.1007/s00431-009-1115-9) contains supplementary material, which is available to authorized users.
\end{abstract}

Hunter, Lücke, and Spranger contributed equally to this work.

K. B. Hunter · C. F. Boerkoel

Child and Family Research Institute,

Department of Medical Genetics, University of British Columbia,

Vancouver, BC, Canada

T. Lücke

Department of Pediatrics, Hannover Medical School,

Hannover, Germany

\section{J. Spranger}

University Children's Hospital,

Mainz, Germany

\section{S. F. Smithson}

Department of Clinical Genetics, St Michael's Hospital,

Bristol, UK

\section{H. Alpay}

Pediatric Nephrology, Marmara University,

Istanbul, Turkey

\section{J.-L. André}

Néphrologie Pédiatrique, Hopital d'Enfants, Centre Hospitalier Universitaire de Nancy,

Vandoeuvre les Nancy Cedex, France

\section{Y. Asakura $\cdot$ N. Stajic}

Department of Endocrinology \& Metabolism,

Kanagawa Children's Medical Center,

Yokohama, Japan in swi/snf-related, matrix-associated, actin-dependent regulator of chromatin, subfamily a-like 1 (SMARCAL1) are the only identified cause of SIOD, but approximately half of patients referred for molecular studies do not have

R. Bogdanovic

Institute of Mother and Child Health Care of Serbia, Belgrade, Serbia

D. Bonneau

Service de Genetique Medicale,

Centre Hospitalier Universitaire d'Angers,

Angers, France

R. Cairns

Department of Radiology, University of British Columbia,

Vancouver, BC, Canada

K. Cransberg

Department of Pediatric Nephrology, Erasmus MC-Sophia, Rotterdam, The Netherlands

S. Fründ

KfH Kinderdialyse,

Münster, Germany

H. Fryssira

Department of Medical Genetics, "Aghia Sophia"

Children's Hospital, Athens University Medical School,

Athens, Greece

D. Goodman

Department of Pathology, School of Medicine,

University of North Carolina,

Chapel Hill, NC, USA 
detectable mutations in SMARCAL1. We hypothesized that skeletal features distinguish between those with or without SMARCAL1 mutations. Therefore, we analyzed the skeletal radiographs of 22 patients with and 11 without detectable SMARCAL1 mutations. We found that patients with SMARCAL1 mutations have a spondyloepiphyseal dysplasia (SED) essentially limited to the spine, pelvis, capital femoral epiphyses, and possibly the sella turcica, whereas the hands and other long bones are basically normal. Additionally, we found that several of the adolescent and young adult patients developed osteoporosis and coxarthrosis. Of the 11 patients without detectable SMARCAL1 mutations, seven had a SED indistinguishable from patients with SMARCAL1 mutations. We conclude therefore that SED is a feature of patients with SMARCAL1 mutations and that skeletal features do not distinguish who of those with SED have SMARCAL1 mutations.

Keywords Genocopy Immunodeficiency · Proteinuria · Skeletal dysplasia $\cdot$ Locus heterogeneity $\cdot$ Schimke immunoosseous dysplasia

$\begin{array}{ll}\text { Abbreviations } & \\ \text { DNA } & \text { Deoxyribonucleic acid } \\ \text { mRNA } & \text { Messenger ribonucleic acid }\end{array}$

K. Helmke

Department of Pediatric Radiology, University of Hamburg, Hamburg, Germany

B. Hinkelmann

Karolinska University Hospital,

Stockholm, Sweden

G. Lama

Department of Pediatrics, University of Naples,

Naples, Italy

P. Lamfers

Mercy Pediatrics and Adolescent Clinic,

Clear Lake, IA, USA

C. Loirat

Pediatric Nephrology, Assistance Publique-Hopitaux de Paris, Hopital Robert Debre,

Paris, France

S. Majore

Medical Genetics, Department of Experimental

Medicine and Pathology, S. Camillo-Forlanini Hospital,

University La Sapienza,

Rome, Italy

C. Mayfield

Southcrest Family and Maternity Care,

Tulsa, OK, USA

B. F. Pontz

Children's Hospital, Technical University,

Munich, Germany
SD

SED

SIOD

SMARCAL1

SNF2

TIA

\section{Introduction}

The osteochondrodysplasias are a heterogeneous group of inherited disorders of skeletal growth and development [28]. Among these, the spondyloepiphyseal dysplasias (SEDs) are characterized by primary involvement of the vertebrae and proximal epiphyseal centers resulting in a short-trunk disproportionate dwarfism $[16,26]$. The radiographic findings, which are frequently age dependent, commonly include flattened vertebrae (platyspondyly) and dysplastic femoral epiphyses.

Schimke immunoosseous dysplasia (SIOD; OMIM \#242900) is an autosomal recessive, pleiotropic disorder

C. Rusu

Medical Genetics Centre, St. Mary Hospital of Iasi, Iasi, Romania

J. M. Saraiva

Consulta de Genética, Hospital Pediátrico de Coimbra,

Coimbra, Portugal

B. Schmidt

Children's Hospital, University of Cologne,

Cologne, Germany

L. Shoemaker

Division of Nephrology, Department of Pediatrics, Kosair

Children's Hospital, School of Medicine, University of Louisville,

Louisville, KY, USA

\section{S. Sigaudy}

Département de Génétique Médicale, Hôpital Timone Enfant, Marseille, France

D. Taha

Department of Pediatrics, King Faisal Specialist

Hospital and Research Centre-Jeddah,

Jeddah, Saudi Arabia

C. F. Boerkoel $(\bowtie)$

Department of Medical Genetics,

Children's and Women's Health Centre of BC,

4500 Oak St., Rm. C234,

Vancouver, BC, Canada V6H 3N1

e-mail: boerkoel@interchange.ubc.ca 
with distinct spondyloepiphyseal abnormalities [27]. Nonskeletal manifestations include mild facial anomalies [3, 23], T cell immunodeficiency [3, 27], nephrotic syndrome [3, 10, 11, 24, 27], hypothyroidism [3], migraine-like headaches [15], cerebral ischemia [10,25], and enteropathy $[13,14,17,18]$.

SIOD is caused by biallelic loss-of-function mutations in SMARCAL1 (OMIM \#606622) [4]. SMARCAL1 encodes a protein homologous to the sucrose nonfermenting type 2 family of chromatin-remodeling proteins [8, 19]; it functions as a DNA annealing helicase [30]. However, in our experience, nearly half of patients with manifestations of SIOD do not have identifiable mutations in the coding exons of SMARCAL1 or detectable alterations of SMARCAL1 mRNA or protein [7] suggesting a close phenotypic overlap with other conditions or genetic heterogeneity.

Table 1 Features of SIOD patients with and without SMARCAL1 mutations

SED spondyloepiphyseal dysplasia, TIA transient ischemic attack
Despite the clinical similarity of these patients, we hypothesized that individuals with and without SMARCAL1 mutations are distinguishable by the type of bone dysplasia. Therefore, we analyzed detailed clinical and radiographic information from a series of 33 SIOD patients with or without detectable SMARCAL1 mutations.

\section{Materials and methods}

Human subjects

Patients referred to this study gave informed consent approved by the Institutional Review Board of the Hospital for Sick Children (Toronto, ON, Canada), Baylor College of Medicine (Houston, TX, USA), or the University of British Columbia (Vancouver, BC, Canada).

\begin{tabular}{|c|c|c|c|c|}
\hline & Dysmorphism & & & \\
\hline & Hair hypoplasia & $11 / 20$ & $5 / 11$ & 0.45 \\
\hline & Broad low nasal bridge & $12 / 21$ & $7 / 11$ & 0.51 \\
\hline & Bulbous nasal tip & $14 / 21$ & $6 / 10$ & 0.29 \\
\hline & Hyperpigmented macules & $14 / 20$ & $6 / 11$ & 0.89 \\
\hline & Protuberant abdomen & $15 / 21$ & $9 / 10$ & 0.25 \\
\hline & Elongated upper lip & $8 / 19$ & $3 / 8$ & 0.59 \\
\hline & Development & & & \\
\hline & Delayed development & $6 / 22$ & $5 / 10$ & 0.15 \\
\hline & Schooling delay & $4 / 11$ & $3 / 7$ & 0.78 \\
\hline & Endocrine & & & \\
\hline & Serologic hypothyroidism & $9 / 19$ & $4 / 9$ & 0.60 \\
\hline & Hematology and immunology & & & \\
\hline & Lymphopenia & $15 / 21$ & $8 / 10$ & 0.48 \\
\hline & Recurrent infections & $9 / 22$ & $6 / 11$ & 0.35 \\
\hline & Neutropenia & $4 / 18$ & $5 / 11$ & 0.18 \\
\hline & Anemia & $10 / 21$ & $6 / 11$ & 0.50 \\
\hline & Thrombocytopenia & $3 / 20$ & $3 / 11$ & 0.26 \\
\hline & Nephrology & & & \\
\hline & Hypertension & $18 / 21$ & $9 / 10$ & 0.61 \\
\hline & Nephrotic syndrome & $22 / 22$ & $9 / 11$ & 0.10 \\
\hline & Progressive renal failure & $13 / 20$ & $8 / 11$ & 0.49 \\
\hline & Proteinuria & $22 / 22$ & $10 / 10$ & 1.0 \\
\hline & Dialysis or graft & $13 / 22$ & $6 / 10$ & 0.64 \\
\hline & Neurology & & & \\
\hline & TIAs & $9 / 22$ & $3 / 10$ & 0.43 \\
\hline & Strokes & $7 / 22$ & $3 / 11$ & 0.56 \\
\hline & Migraine-like headaches & $8 / 17$ & $1 / 6$ & 0.21 \\
\hline SED spondyloepiphyseal & Skeletal radiographic findings & & & \\
\hline dysplasia, TIA transient & SED & $22 / 22$ & $7 / 11$ & 0.01 \\
\hline
\end{tabular}


Analysis of clinical features

The clinical data for patients were obtained from questionnaires completed by the referring physician as well as medical records and summaries provided by that physician. The data obtained for patients with and without SMARCAL1 mutations were tabulated and then summed to allow comparison of the features of patients in each group. If a feature was not reported for a patient, then that patient was excluded from the denominator. Statistically significant differences between the groups were determined by the Fisher's exact test. Also, to subgroup patients according to disease severity, each patient's signs and symptoms were scored as previously described [12].

Analysis of skeletal radiographs

All radiographs were reviewed independently by Jürgen Spranger and Cornelius F. Boerkoel.

\section{Results}

Since we had previously found that only about half of patients clinically diagnosed with SIOD have detectable SMARCAL1 mutations, we sought to identify skeletal radiographic features distinguishing these two groups of patients [7]. Identification of such features would be useful for guiding molecular diagnosis as well as for characterization of other genetic causes of SIOD. To this end, we assembled detailed clinical and skeletal radiographic data on 33 patients, 11 without SMARCAL1 mutations and 22 with biallelic SMARCAL1 mutations.

\section{Phenotypic comparisons}

The 22 patients with and 11 without detectable SMARCAL1 mutations have very similar clinical features (Table 1; Supplementary Tables 1 and 2) and similar disease severity scores, which we calculated as previously described [7]. Those with SMARCAL1 mutations have slightly more neurological complications, but these are insufficient to distinguish between the two groups of patients. Given the inadequacy of clinical features for distinguishing those with and without SMARCAL1 mutations, we looked for distinguishing radiographic features.

\section{Radiographic findings}

As previously described [26], the spondyloepiphyseal dysplasia of SIOD is most prominent in the spine, pelvis,

Fig. 1 Typical bony features of SIOD. a Lateral spine radiograph of a 5-year-old child showing dorsally flattened, pear-shaped vertebral bodies. b Lateral skull radiograph of a 5-year-old child showing the typical widening of the sella. c Posteroanterior hand radiograph of a 13 -year-old adolescent showing the absence of bony abnormalities. $\mathbf{d}$ Anteroposterior hip radiograph of a 4-year-old child showing the small, laterally displaced capital femoral epiphyses, hypoplastic basilar ilia, and upslanting and poorly formed acetabula

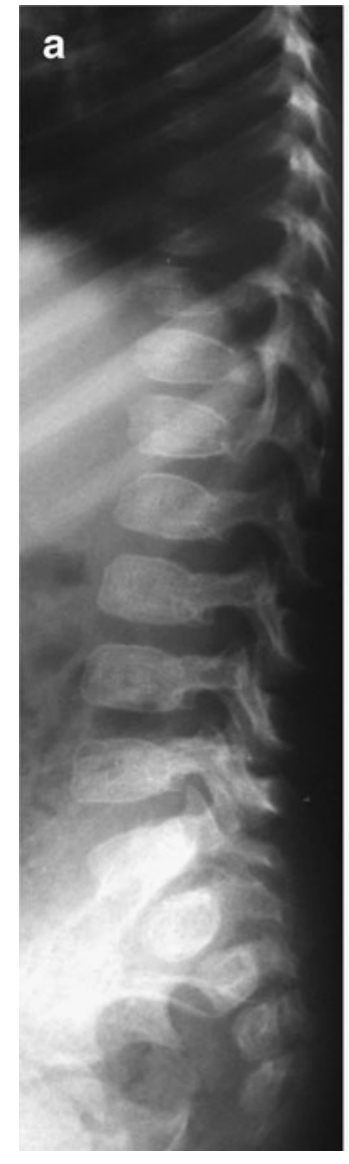

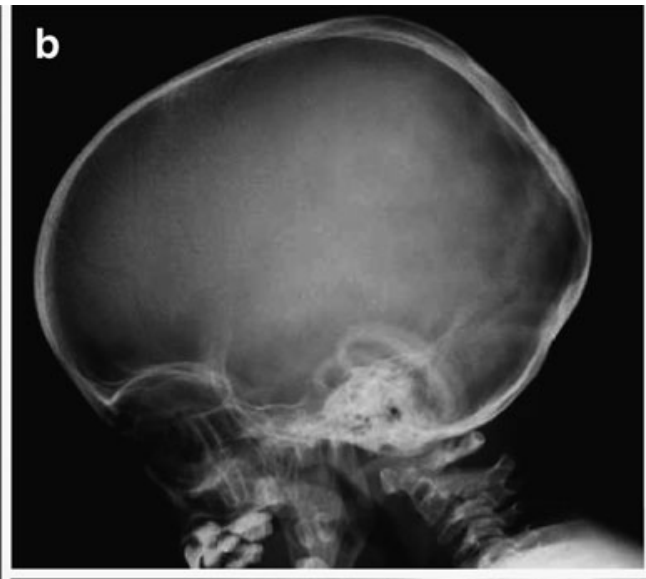
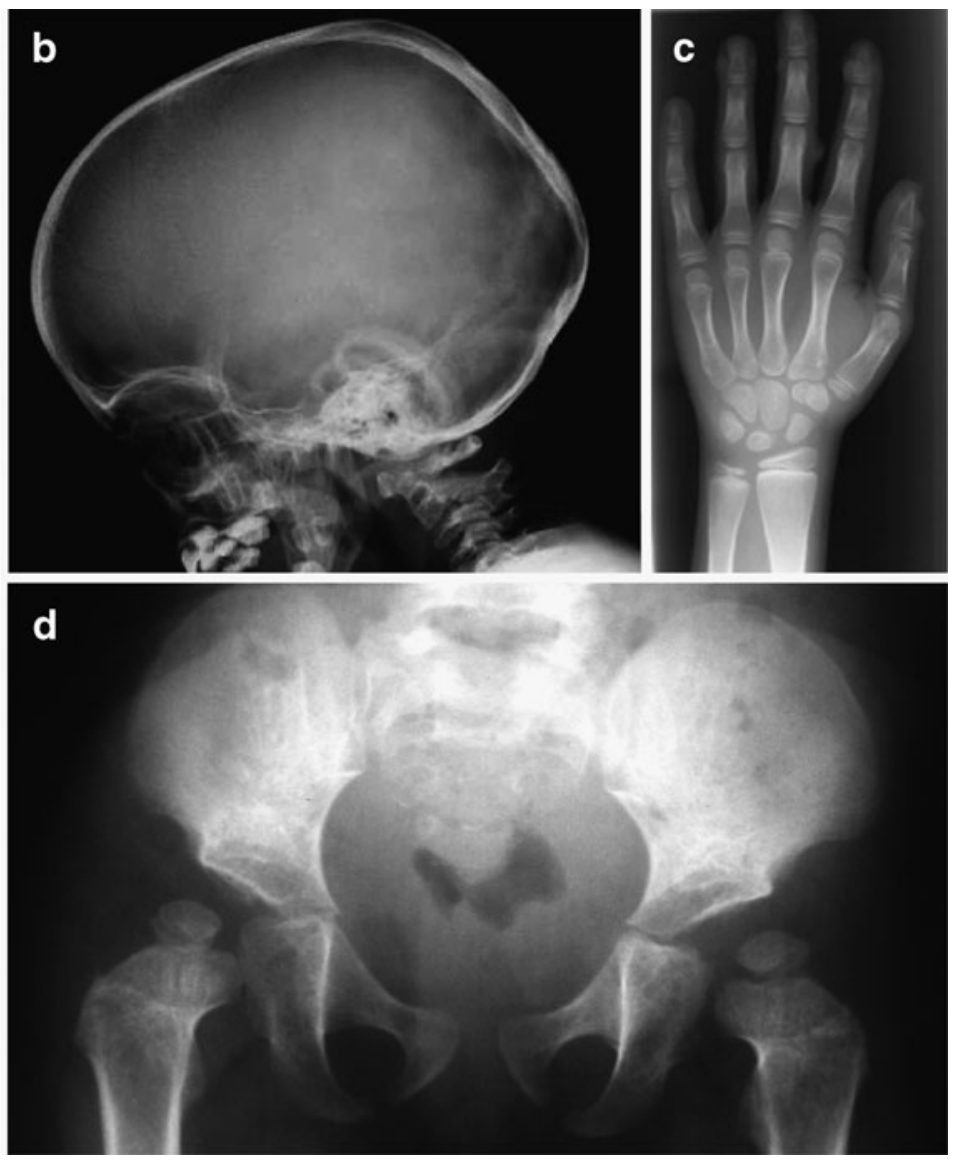
and capital femoral epiphyses (Fig. 1). The hands and other long bones are essentially normal.

\section{Spine findings among patients with SMARCAL1 mutations}

The vertebral bodies are ovoid, dorsally flattened and without segmentation defects at all ages (Figs. 2 and 3). The spinal changes between 4 and 6 years of age show dorsally flattened, pear-shaped vertebral bodies. By 7 11 years of age, there is more generalized vertebral flattening with slightly irregular upper and lower plates (see SD114, Fig. 2). There is variable expressivity as only mild abnormalities are observed in some patients (see SD112, Fig. 2). By adulthood, all have clear signs of progressive osteopenia with compressed vertebral bodies and vertebral bone density similar to that of the soft tissue (Figs. 1 and 2). Peculiarly, SD27 also has diffuse calcification of her discs (Figs. 2 and 3).

Pelvis and capital femoral epiphyseal findings among patients with SMARCAL1 mutations

Nearly consistently at all ages, the pelvis and femora are notable for small, laterally displaced capital femoral

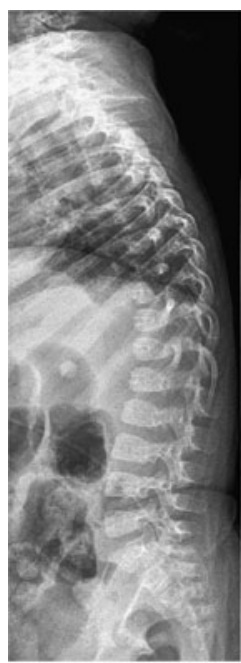

3 years

(SD121)

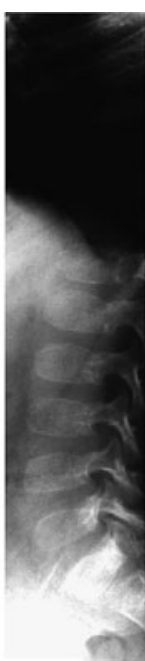

4 years

(SD70)

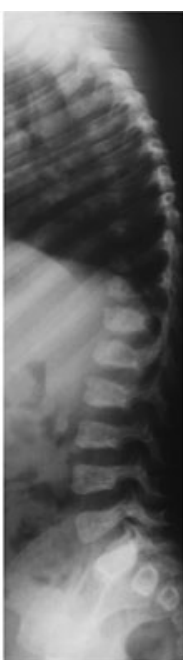

4 years

(SD50)

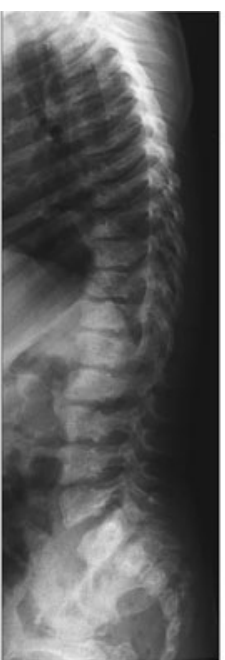

5 years

(SD79)

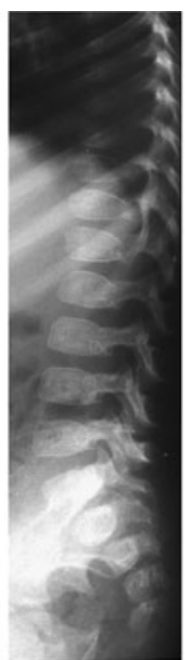

5 years

(SD44)

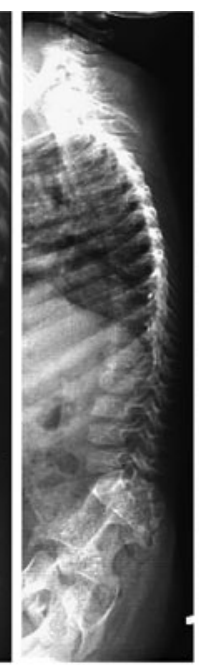

5 years

(SD61)

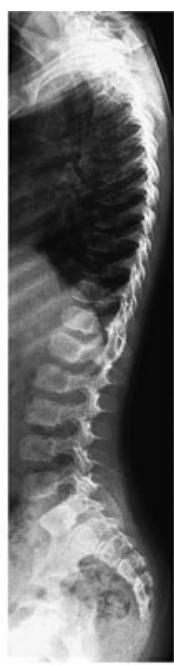

5 years

(SD78)

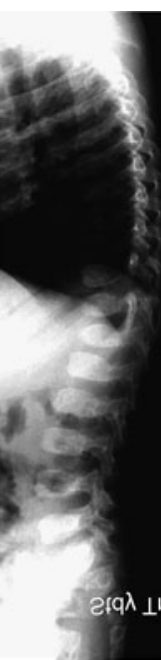

6 years

(SD66)

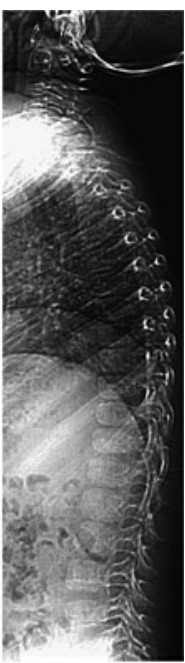

7 years

(SD38)

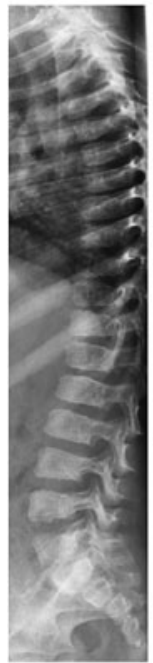

7 years

(SD101)

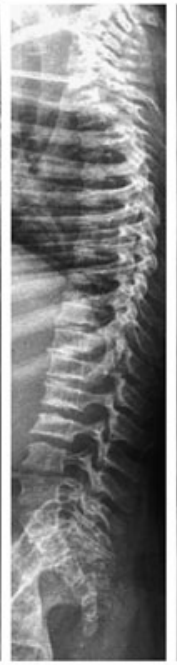

8 years

(SD114)

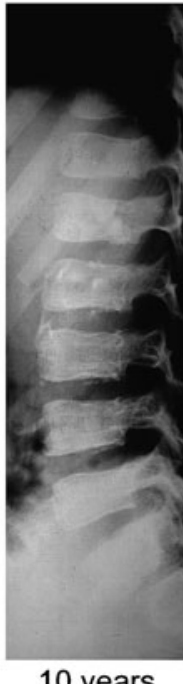

10 years

(SD39)

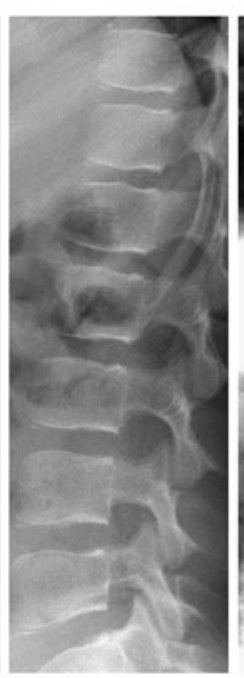

11 years

(SD112)

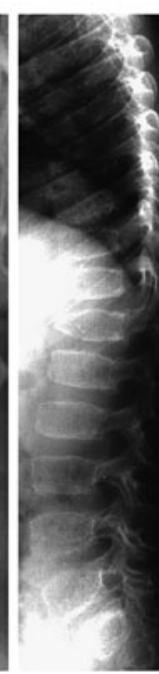

11 years (SD66)

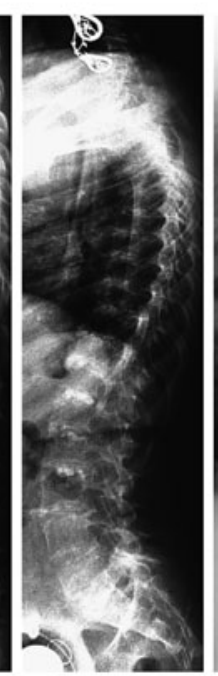

23 years

(SD27)

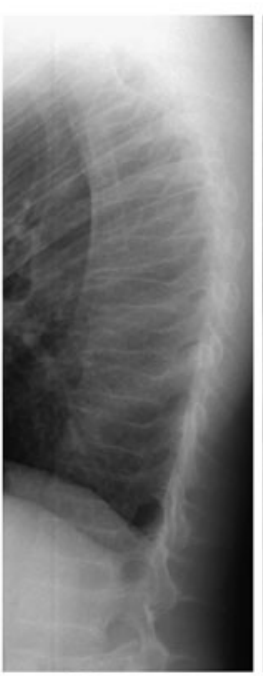

23 years

(SD65)

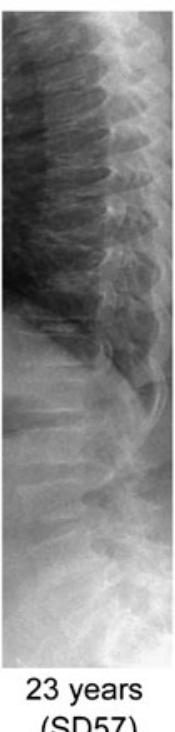

Fig. 2 Lateral spine radiographs of patients with identified SMARCAL1 mutations at different ages. The vertebral bodies are flattened at all ages and pear-shaped between 4 and 6 years. There is generalized vertebral flattening with slightly irregular upper and lower plates in some patients. The severity of the vertebral changes is variable as illustrated by comparison of SD112 with SD66. Note the radiolucency of adult vertebrae consistent with the progressive osteopenia of SIOD patients. SD27 had diffuse disc calcification; the significance of this is uncertain 

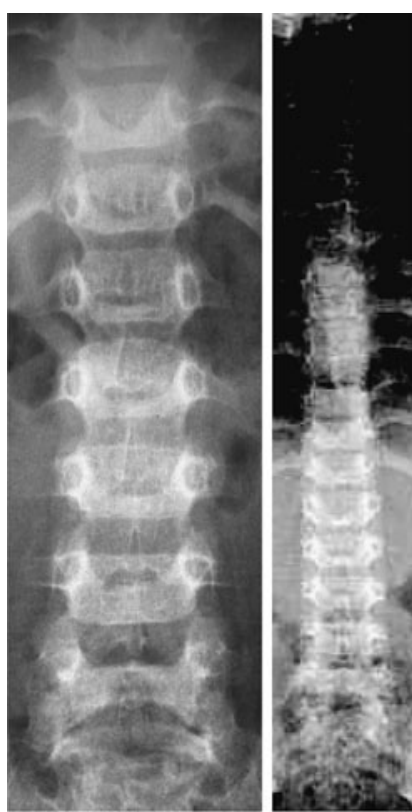

4 years

(SD96)

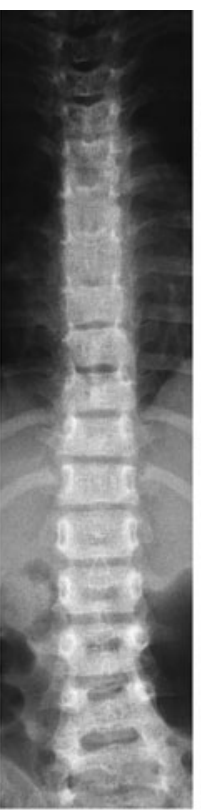

5 years

(SD79)

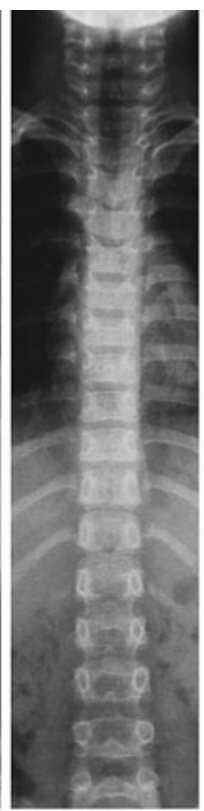

5 years

(SD78)

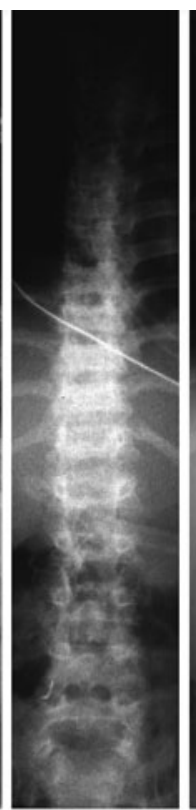

8 years (SD50)

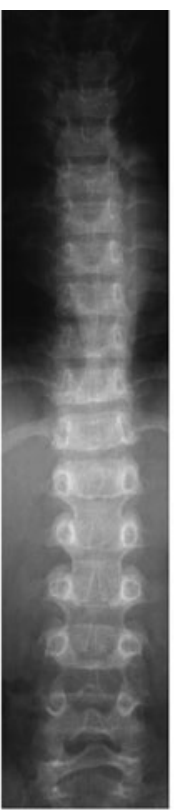

8 years

(SD66)

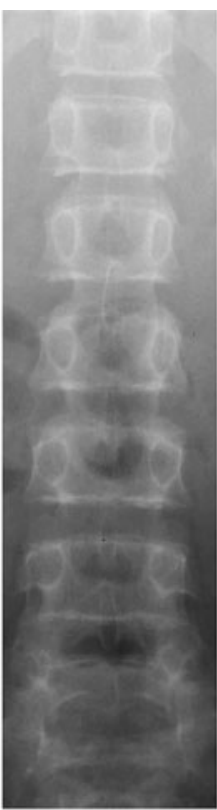

12 years (SD113)
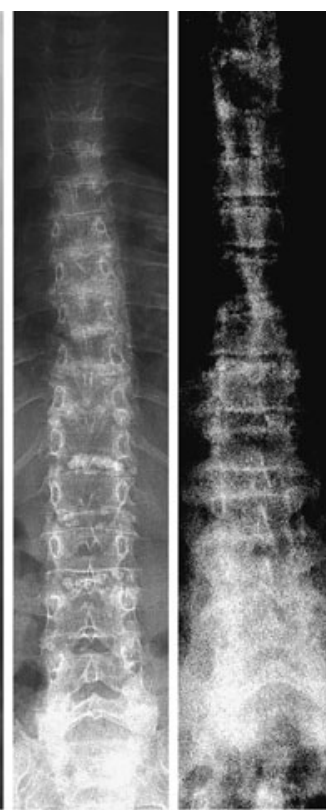

23 years 23 years

(SD27)

(SD57)

Fig. 3 Anterior-posterior spine radiographs of patients with identified SMARCAL1 mutations at different ages show various degrees of platyspondyly. SD27 had diffuse disc calcification; the significance of this is uncertain

epiphyses, hypoplastic basilar ilia with upslanting and poorly formed acetabula (Fig. 4). The femoral dysplasia progresses to premature coxarthrosis requiring prosthetic therapy (SD27, Fig. 4).

The severity of these skeletal changes varies among the patients. For example, the proximal femoral pathology ranges from coxa valga with well-preserved capital femoral epiphyses and a normal pelvis (SD78, Fig. 4) to short femoral necks with a peculiar lip-like medial protrusion and markedly hypoplastic lower ilia (SD121, Fig. 4).

\section{Skull findings among patients with SMARCAL1 mutations}

No bony abnormalities have been reported in the skull of SIOD patients before. However, of five skull radiographs, three (SD44, SD61, SD114) show a markedly wide sella turcica, one (SD120) a depression of the anterior portion of the sella, and one (SD79) a normal sella turcica (Fig. 5).

\section{Hand and feet findings among patients with SMARCAL1 mutations}

Although one SIOD individual has been referred to us with preaxial hexadactyly (C. F. Boerkoel, unpublished data), no others have been reported with bony abnormalities of the hands or feet [26]. Consistent with this, the hand and feet radiographs from the patients in this study do not show bony abnormalities (Fig. 5).

\section{Skeletal findings among patients without detectable SMARCAL1 mutations}

Among the patients without detectable SMARCAL1 mutations, three (SD87, SD95, SD55) have skeletal abnormalities typical of SIOD (Fig. 6). Two (SD80, SD85) have a mild spondyloepiphyseal dysplasia consistent with SIOD (Fig. 6), similar to that of patient SD112 (Figs. 2, 3, and 4). One (SD54) has a typical SIOD-type spondyloepiphyseal dysplasia and severe scoliosis and anterior hypoplasia of L2; these latter changes, which have not been observed among SIOD patients with a SMARCAL1 mutation, may be secondary to an unrelated muscular hypotonia.

\section{Patients distinct from SIOD}

Radiographs from four patients diagnosed with SIOD but without a detectable SMARCAL1 mutation do not show the

Fig. 4 Hip radiographs of patients with identified SMARCAL1 mutations at different ages. The femora generally have small, laterally displaced capital epiphyses, but normal epiphyseal ossification may occur (e.g., SD78, SD 112). By adulthood, the femoral dysplasia usually progresses to premature coxarthrosis (SD18 and SD27) requiring prosthetic therapy (SD27). The ilia are usually small because of hypoplastic basilar portions and have upslanting and poorly formed acetabula. The severity of the ilia and femoral changes is variable as illustrated by comparison of SD78, who had preserved capital femoral epiphyses and a normal pelvis, with SD121, who had short femoral necks with a peculiar lip-like medial protrusion and markedly hypoplastic basilar ilia 


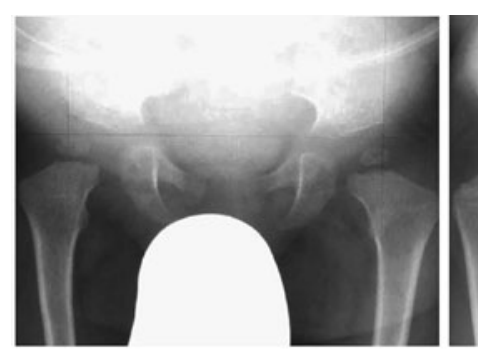

2.5 years (SD33)

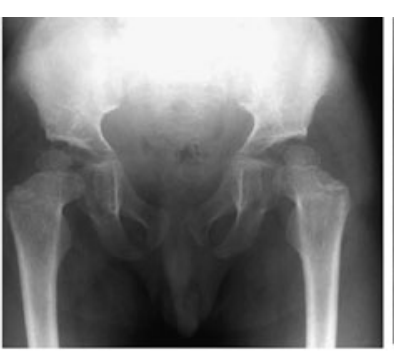

3 years (SD74)

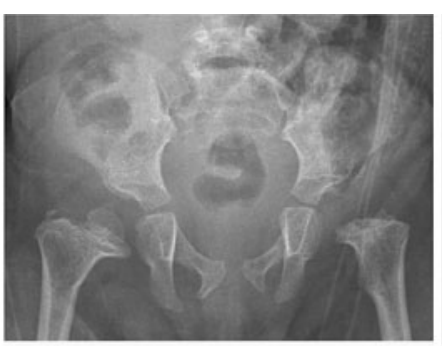

3 years (SD121)

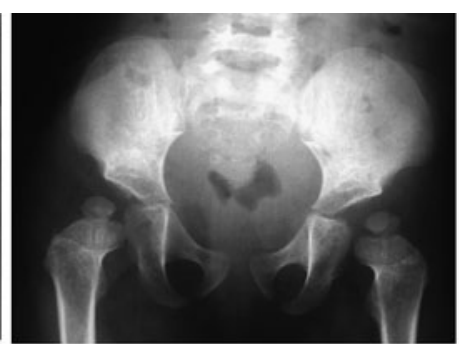

4 years (SD50)

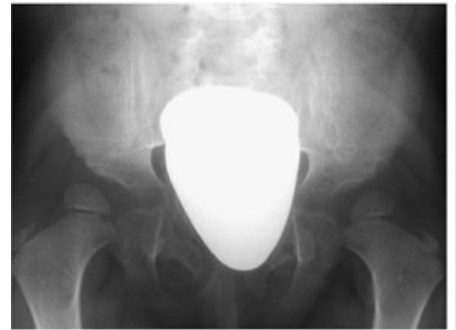

4 years (SD70)

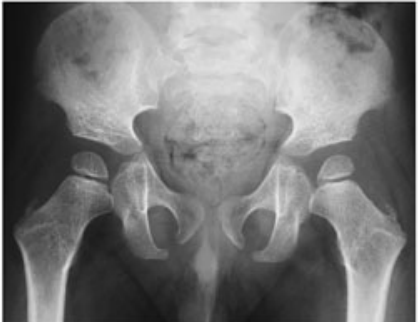

4 years (SD96)

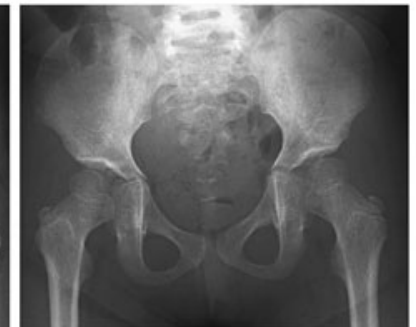

4.5 years (SD79)

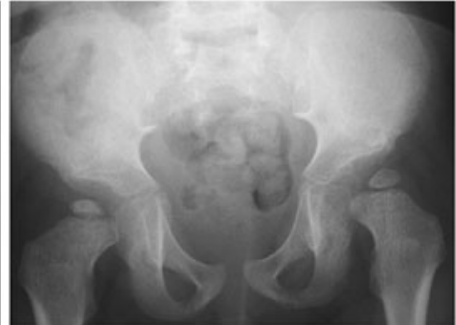

5 years (SD44)

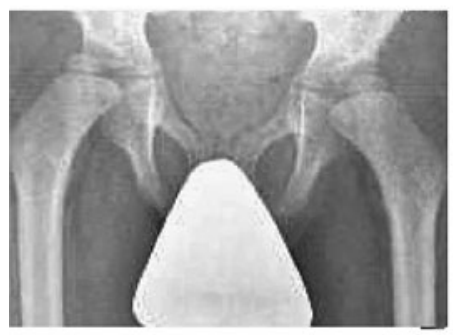

5 years (SD61)

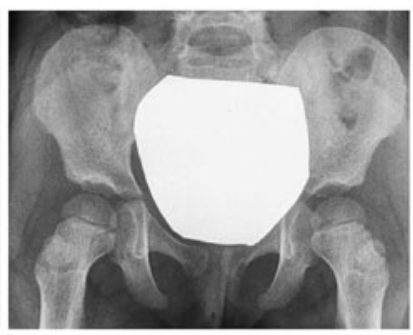

5 years (SD78)

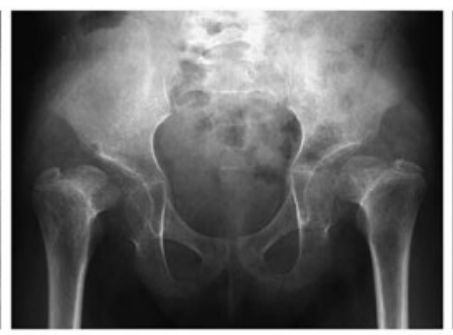

6 years (SD66)

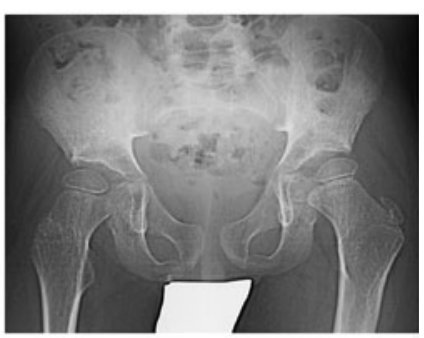

7 years (SD38)

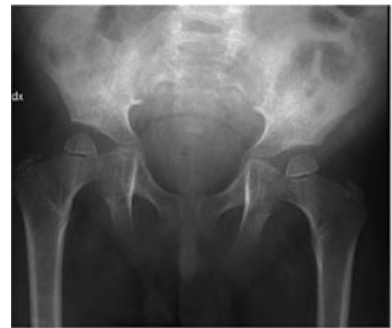

7 years (SD101)

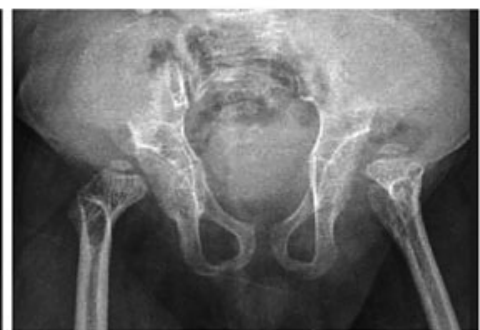

8 years (SD114)

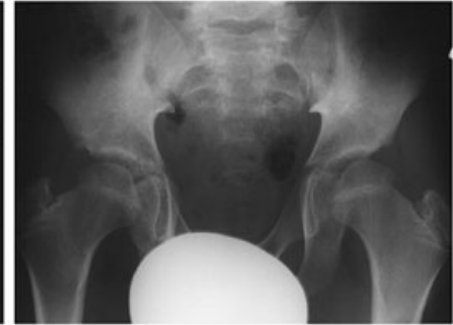

10 years (SD39)

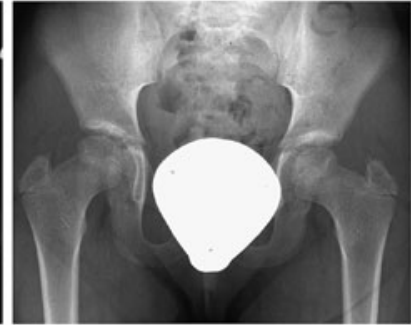

11 years (SD112)

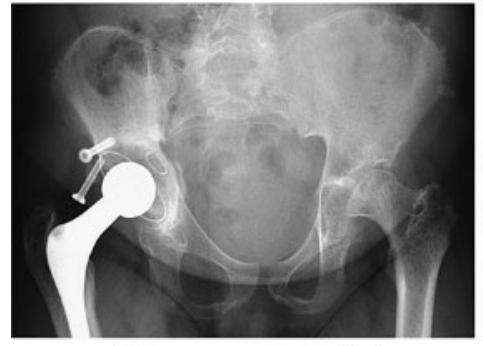

25 years (SD27)

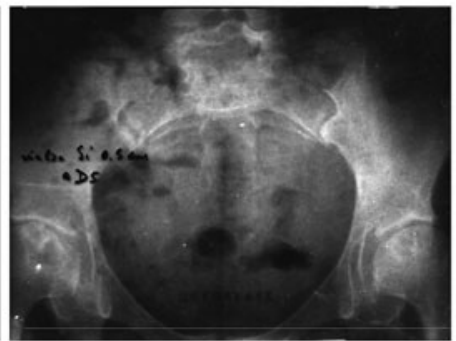

adult (SD18) 


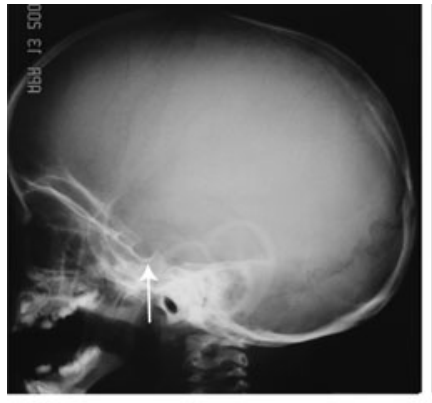

1.8 years

(SD120)

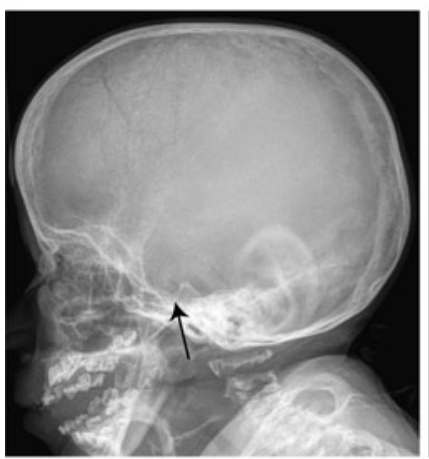

8 years

(SD114)

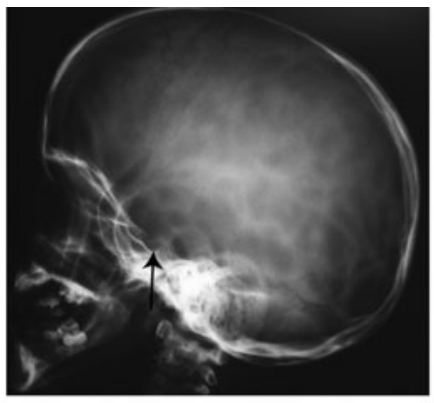

5 years

(SD44)

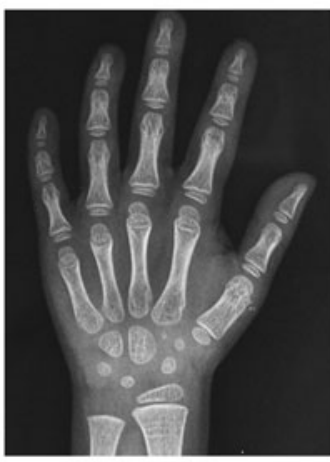

5 years

(SD78)

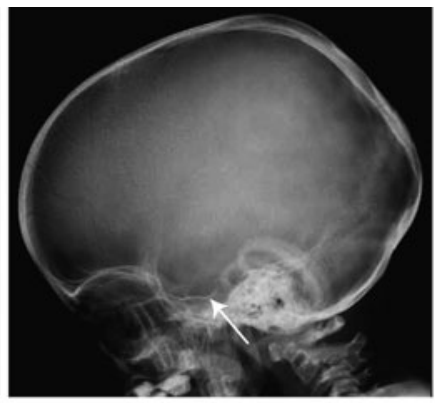

5 years

(SD61)

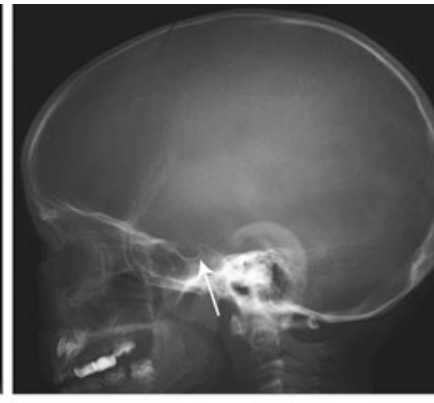

5 years

(SD79)

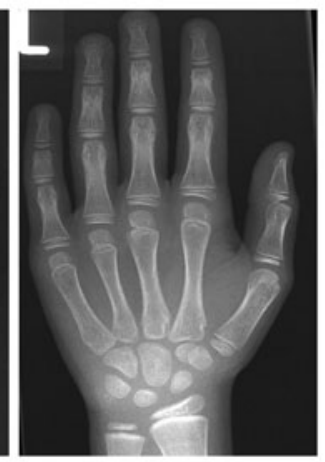

12 years

(SD113)

Fig. 5 Skull and hand radiographs of patients with identified SMARCAL1 mutations at different ages. Note the marked widening of the sella in SD44, SD61, and SD79 and mild widening of the sella in SD120. No bony abnormalities are observed in the hands

skeletal features consistent with SIOD. The radiographs of one (SD89) show enchondromata in the tubular and flat bones and flattened vertebral bodies with irregular areas of increased and decreased mineralization (data not shown); these findings are most consistent with a form of spondyloenchondrodysplasia [21]. The radiographs from three (SD81, SD52a, SD52b) have no signs of SED.

\section{Discussion}

The characteristic skeletal features of SIOD patients with SMARCAL1 mutations are (1) ovoid and flattened vertebral bodies without segmentation defects, (2) small, laterally displaced capital femoral epiphyses, hypoplastic basilar ilia, and upslanting and poorly formed acetabula, and (3) possibly a wide sella. The flattening of the vertebrae and the abnormalities of the hips usually worsen with age and do so to a degree independent of the severity of other features of SIOD. Also, during later childhood, adolescence, and early adulthood, many individuals with SIOD develop coxarthrosis and vertebral osteopenia.

A wide sella turcica has not been reported among SIOD patients previously. If additional studies confirm the findings in this small number of patients, then this will be an additional marker for the clinical diagnosis of SIOD. The wide sella turcica does not reflect anterior pituitary or adenohypophysis dysfunction since in the three patients tested, all had normal growth hormone and thyroid stimulating hormone levels. Also, prior reviews of anterior pituitary function in SIOD patients have not identified a functional pituitary defect [3].

One reason the wide sella turcica may not have been noted previously is the high degree of intra- and interfamilial variability of SIOD $[2,7]$. This is reinforced by our findings that not all SIOD patients with SMARCAL1 mutations had each of the typical skeletal findings. Additionally, we found that there was even variability among tissues within the same person since the severity of vertebral flattening did not predict the severity of hip dysplasia or vice versa.

Relevant to our question of whether the clinical skeletal radiographs can guide molecular testing, our results show that none of the patients without SED had detectable SMARCAL1 mutations. Therefore, testing for SMARCAL1 mutations in this group may not be indicated although studies with more patients are needed to confirm this. On the other hand, for individuals with radiographic findings of SED, there were no distinguishing radiographic or clinical features to suggest who would or would not have detectable 


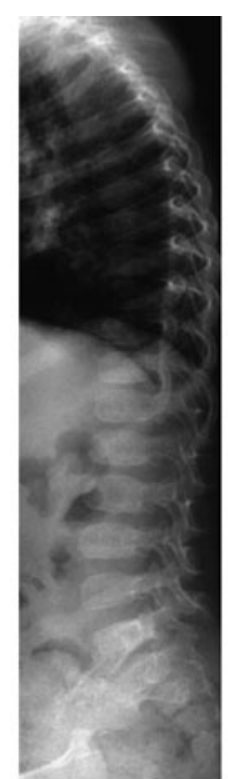

4 years

(SD95)

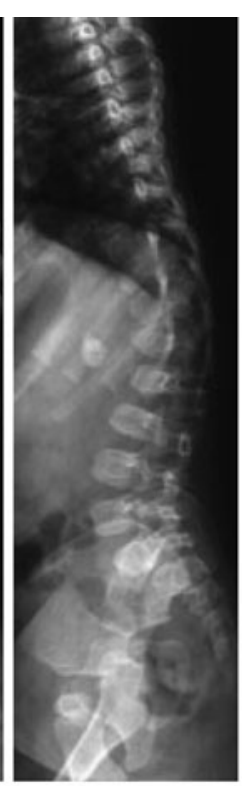

5 years

(SD54)

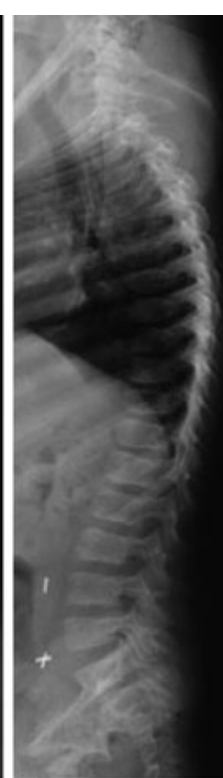

5 years

(SD80)

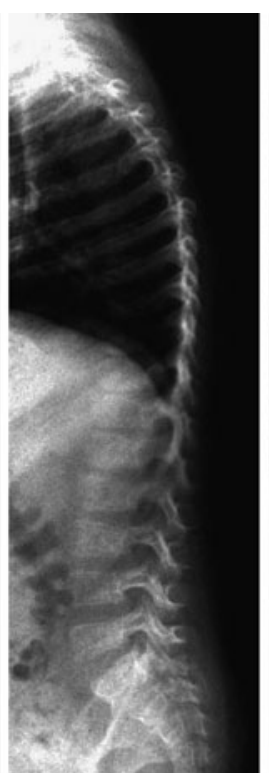

5 years

(SD87)

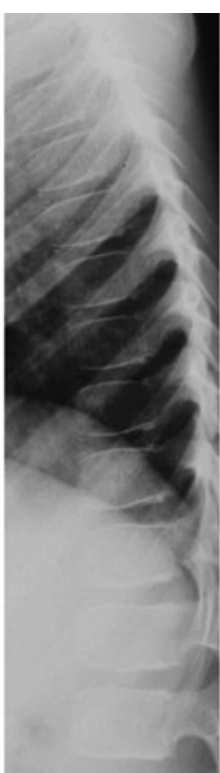

6 years (SD55)

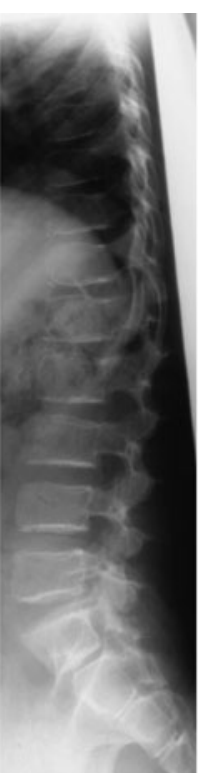

20 years

(SD85)

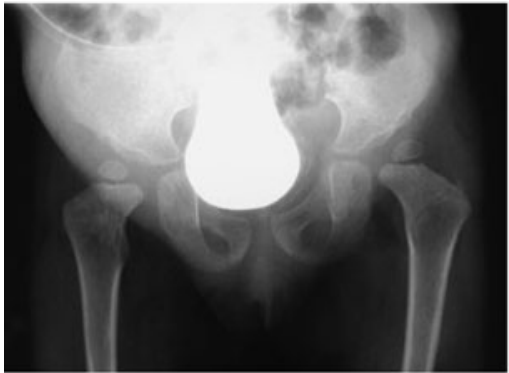

4 years (SD95)

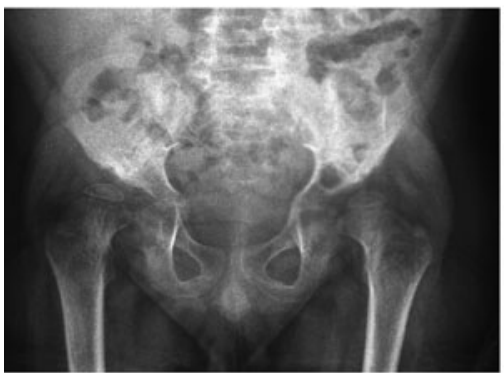

5 years (SD87)

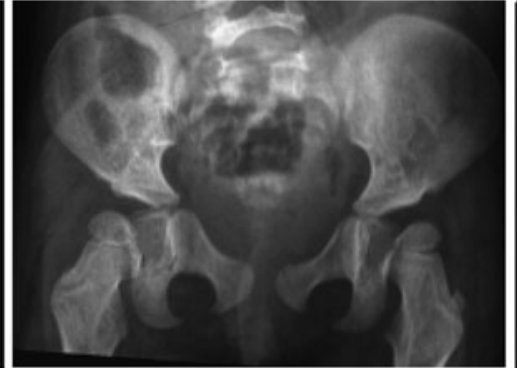

5 years (SD54)

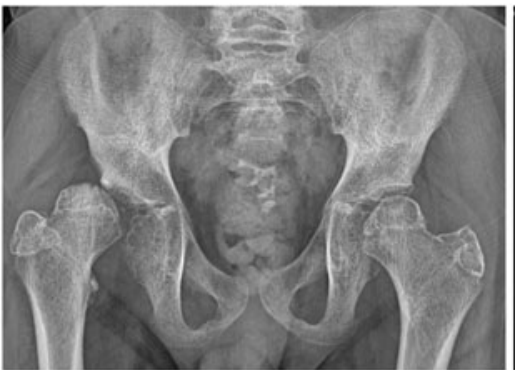

12 years (SD55)

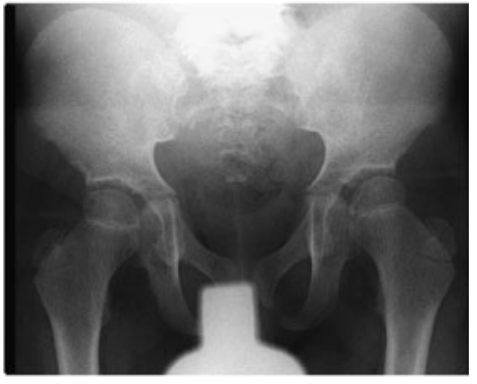

5 years (SD80)

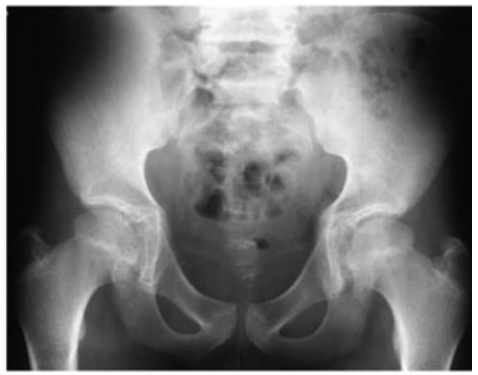

20 years (SD85)
Fig. 6 Lateral spine and hip radiographs of SIOD patients without detectable SMARCAL1 mutations at different ages. Note the similarity of bony features to those with SMARCAL1 mutations. SD54 had

SMARCAL1 mutations. Therefore, all patients with SED and the other clinical signs of SIOD should be tested for SMARCAL1 mutations.

The known molecular mechanism underlying SIOD is a generalized disturbance of genomic structure arising secondary to loss of SMARCAL1 DNA strand annealing [31]. This disturbance disrupts both DNA replication $[1,6,9,20$, 29, 31] and RNA transcription (Baradaran-Heravi et al., kyphoscoliosis and anterior hypoplasia of L2, which may have been secondary to an unrelated muscular hypotonia

submitted for publication; Morimoto et al., submitted for publication). Since cell proliferation and RNA transcription are quantitative traits affected by environment, stochastics, and genetic background [5, 22], the variability among patients and tissues can be accounted for by the combined impact of environment, stochastics, and genetic variation.

The similar clinical and radiographic features of individuals with and without SMARCAL1 mutations also 
suggest that SIOD could be induced by some environmental conditions or by mutations in genes other than SMARCAL1. Evidence supporting genetic heterogeneity as opposed to environmental factors includes the recurrence of disease in siblings, the absence of disease in parents, and the geographic dispersal of patients. Of interest for human biology, this suggests that although SMARCAL1 is the only identified annealing helicase in humans [30], other enzymes with redundant or similar function may exist or that SMARCAL1 deficiency mimics that of another global modulator of chromatin structure. Assessments of these possibilities as well as delineation of the physiologic mechanism leading to the skeletal abnormalities will require studies in model organisms.

Besides being unable to distinguish SIOD patients with and without SMARCAL1 mutations, skeletal radiographs also do not differentiate SIOD from many other spondyloepiphyseal dysplasias. The differential diagnosis of SIOD includes other forms of spondyloepiphyseal and spondyloepimetaphyseal dysplasia listed in group 11 of the International Nosology [28]. These are distinguished from SIOD by their different clinical presentation and by the absence of the characteristic extraskeletal features such as facial dysmorphism, skin changes, $\mathrm{T}$ cell deficiency, and renal failure. Thus, the skeletal features are not pathognomonic of SIOD.

In summary, we further define the radiographic features of SIOD and show that SED is a feature common to all individuals with SIOD and SMARCAL1 mutations. However, among SIOD patients with SED, no radiographic features distinguish those with SMARCAL1 mutations from those without SMARCAL1 mutations. Understanding of the clinical variability of this disorder as well as other possible genetic causes requires further elucidation of the molecular mechanisms underlying SIOD.

Acknowledgements The authors thank Drs. Alireza BaradaranHeravi, Jan M. Friedman, and Judith Hall for critical review of this manuscript. This work was supported in part by grants from the Child \& Family Research Institute (C. F. Boerkoel) and the Michael Smith Foundation for Health Research (C. F. Boerkoel).

Conflict of interest The authors declare no conflicts of interest.

Open Access This article is distributed under the terms of the Creative Commons Attribution Noncommercial License which permits any noncommercial use, distribution, and reproduction in any medium, provided the original author(s) and source are credited.

\section{References}

1. Bansbach CE, Betous R, Lovejoy CA et al (2009) The annealing helicase SMARCAL1 maintains genome integrity at stalled replication forks. Genes Dev 23:2405-2414
2. Baradaran-Heravi A, Thiel C, Rauch A et al (2008) Clinical and genetic distinction of Schimke immuno-osseous dysplasia and cartilage-hair hypoplasia. Am J Med Genet A 146A:20132017

3. Boerkoel CF, O’Neill S, Andre JL et al (2000) Manifestations and treatment of Schimke immuno-osseous dysplasia: 14 new cases and a review of the literature. Eur J Pediatr 159:1-7

4. Boerkoel CF, Takashima H, John J et al (2002) Mutant chromatin remodeling protein SMARCAL1 causes Schimke immuno-osseous dysplasia. Nat Genet 30:215-220

5. Cheung VG, Spielman RS (2009) Genetics of human gene expression: mapping DNA variants that influence gene expression. Nat Rev Genet 10:595-604

6. Ciccia A, Bredemeyer AL, Sowa ME et al (2009) The SIOD disorder protein SMARCAL1 is an RPA-interacting protein involved in replication fork restart. Genes Dev 23:2415-2425

7. Clewing JM, Fryssira H, Goodman D et al (2007) Schimke immunoosseous dysplasia: suggestions of genetic diversity. Hum Mutat 28:273-283

8. Coleman MA, Eisen JA, Mohrenweiser HW (2000) Cloning and characterization of HARP/SMARCAL1: a prokaryotic HepArelated SNF2 helicase protein from human and mouse. Genomics $65: 274-282$

9. Driscoll R, Cimprich KA (2009) HARPing on about the DNA damage response during replication. Genes Dev 23:2359-2365

10. Ehrich JH, Burchert W, Schirg E et al (1995) Steroid resistant nephrotic syndrome associated with spondyloepiphyseal dysplasia, transient ischemic attacks and lymphopenia. Clin Nephrol 43:89-95

11. Ehrich JH, Offner G, Schirg E et al (1990) Association of spondylo-epiphyseal dysplasia with nephrotic syndrome. Pediatr Nephrol 4:117-121

12. Elizondo LI, Cho KS, Zhang W et al (2009) Schimke immunoosseous dysplasia: SMARCAL1 loss-of-function and phenotypic correlation. J Med Genet 46:49-59

13. Hashimoto K, Takeuchi A, Ieshima A et al (1994) Juvenile variant of Schimke immunoosseous dysplasia. Am J Med Genet 49:266269

14. Kaitila I, Savilahti E, Ormala T (1998) Autoimmune enteropathy in Schimke immunoosseous dysplasia. Am J Med Genet 77:427430

15. Kilic SS, Donmez O, Sloan EA et al (2005) Association of migrainelike headaches with Schimke immuno-osseous dysplasia. Am J Med Genet A 135:206-210

16. Lachman RS (2006) Taybi and Lachman's radiology of syndromes, metabolic disorders and skeletal dysplasias, 5th edn. Mosby, Philadelphia

17. Lama G, Marrone N, Majorana M et al (1995) Spondyloepiphyseal dysplasia tarda and nephrotic syndrome in three siblings. Pediatr Nephrol 9:19-23

18. Lücke T, Billing H, Sloan EA et al (2005) Schimke-immunoosseous dysplasia: new mutation with weak genotype-phenotype correlation in siblings. Am J Med Genet A 135:202-205

19. Muthuswami R, Truman PA, Mesner LD, Hockensmith JW (2000) A eukaryotic SWI2/SNF2 domain, an exquisite detector of double-stranded to single-stranded DNA transition elements. J Biol Chem 275:7648-7655

20. Postow L, Woo EM, Chait BT, Funabiki H (2009) Identification of SMARCAL1 as a component of the DNA damage response. J Biol Chem. doi:10.1074/jbc.M109.048330

21. Renella R, Schaefer E, LeMerrer M et al (2006) Spondyloenchondrodysplasia with spasticity, cerebral calcifications, and immune dysregulation: clinical and radiographic delineation of a pleiotropic disorder. Am J Med Genet A 140:541-550

22. Rockman MV, Kruglyak L (2006) Genetics of global gene expression. Nat Rev Genet 7:862-872 
23. Saraiva JM, Dinis A, Resende C et al (1999) Schimke immunoosseous dysplasia: case report and review of 25 patients. J Med Genet 36:786-789

24. Schimke RN, Horton WA, King CR (1971) Chondroitin-6sulphaturia, defective cellular immunity, and nephrotic syndrome. Lancet 2:1088-1089

25. Schmidt B, Christen HJ, Herkenrath P et al (1997) Cerebral complications in Schimke immuno-osseous dysplasia. Eur J Pediatr 156:789-791

26. Spranger JW, Brill PW, Poznanski A (2002) Bone dysplasias: an atlas of genetic disorders of skeletal development. Oxford University Press, New York
27. Spranger J, Hinkel GK, Stoss H et al (1991) Schimke immunoosseous dysplasia: a newly recognized multisystem disease. J Pediatr 119:64-72

28. Superti-Furga A, Unger S (2007) Nosology and classification of genetic skeletal disorders: 2006 revision. Am J Med Genet A 143:1-18

29. Yuan J, Ghosal G, Chen J (2009) The annealing helicase HARP protects stalled replication forks. Genes Dev 23:2394-2399

30. Yusufzai T, Kadonaga JT (2008) HARP is an ATP-driven annealing helicase. Science 322:748-750

31. Yusufzai T, Kong X, Yokomori K, Kadonaga JT (2009) The annealing helicase HARP is recruited to DNA repair sites via an interaction with RPA. Genes Dev 23:2400-2404 